\title{
ADMINISTRAÇÃO DE ESTOQUES PARA BENS DE VAREJO NÃO PERECÍVEIS
}

\author{
Jorge Luiz de Biazzi \\ Departamento de Administração \\ Faculdade de Economia, Administração e Contabilidade/USP
}

[Este artigo foi baseado na Dissertação de Mestrado do autor, apresentada e aprovada no Departamento de Engenharia de Produção da Escola Politécnica da USP em setembro de 1993]

Este estudo compara métodos de administração de sistemas de estoques de bens de varejo não perecíveis, considerando o caso de perda de venda. Diversos métodos, baseados em modelos com diferentes hipóteses, são testados em ambientes caracterizados por comportamento da demanda e relações entre custos, entre outras características. A análise baseou-se numa simulação que avaliou o comportamento do sistema a cada intervalo de tempo num certo horizonte. O programa utilizado contém módulos para projeção de demanda baseada em suavizamento exponencial, cálculos físicos e econômicos e cálculo dos parâmetros de cada um dos métodos. Os métodos baseados em modelos de revisão periódica e demanda probabilística mostraram-se mais adequados do que os baseados, respectivamente, em revisão contínua e demanda determinística, assim como os que adotam lead-times máximos sobrepujaram os que adotam lead-times médios. Os desempenhos relativos dos métodos não foram afetados por alterações na aleatoriedade da demanda. Os métodos que operam baseados na projeção da demanda de vários períodos à frente mostraram-se mais eficazes do que os demais quando há picos de demanda, embora, nas demais situações (demanda estável, crescente ou decrescente, sem sazonalidade ou com sazonalidade suave no curto prazo), estes métodos sejam equiparáveis aos métodos baseados em revisão periódica e demanda estável ao longo do tempo.

Palavras-chaves: estoques, simulação, administração de materiais, varejo

\section{Introdução}

Este trabalho tem como objeto a administração de estoques de bens de varejo com algumas características específicas intrínsecas e de mercado. Os bens cuja administração é estudada neste trabalho são bens de consumo não perecíveis (ao menos no horizonte de algumas semanas), com demanda significativa e ciclo de vida longo, como bolachas, sabonetes, farinha de trigo, leite condensado, etc.

Os mercados em que tais produtos são considerados são aqueles em que há várias opções para o consumidor final, de forma que, quando um determinado varejista não tem o bem disponível no momento desejado pelo cliente, este pode, sem custos 
significativos, dirigir-se a outro varejista, caracterizando a situação conhecida como perda de venda. Deve-se ressaltar que, neste estudo, não há a distinção entre marcas (os diferentes rótulos que pode ter um mesmo bem, produzido por um ou mais fabricantes).

Nesta situação, normalmente o preço de venda de um bem é determinado não pelas condições operacionais do varejista, mas em função do preço praticado pelo mercado. Desta forma, torna-se imprescindível uma boa administração dos estoques destas empresas, pois os custos associados a estes estão entre os principais componentes dos custos totais das empresas, sendo fator determinante na sua lucratividade.

O presente estudo tem como objetivos: (1) a identificação de métodos de planejamento e controle de sistemas de estoques (procedimentos) que possam ser utilizados pelas empresas varejistas, (2) a elaboração de um método geral para abordagem dos problemas de administração de estoques para os bens de varejo tratados, a partir do qual seriam feitas adaptações para que se pudesse estudar cada situação de mercado em particular, permitindo a indicação do procedimento mais apropriado a cada uma delas e (3) a verificação do comportamento dos procedimentos identificados e selecionados em cada ambiente e, com sua comparação, a obtenção de indicações gerais sobre o seu uso e a sua adequação. Para se atingir os dois últimos objetivos, é utilizado um programa (software) de simulação de sistemas de produção.

As situações de mercado consideradas incluem demanda probabilística (estável ou linearmente crescente ou decrescente, com ou sem sazonalidade), lead-time (intervalo de tempo entre a verificação da necessidade do pedido e a sua utilização na empresa) determinístico ou probabilístico e diferentes relações entre os custos unitários relativos a estoques. O ciclo das sazonalidades será anual, com comportamento "senoidal" (com "picos" e "vales" de demanda) ou tendo picos localizados em um curto intervalo de tempo (Natal, por exemplo).

Os bens são tratados independentemente e não são analisadas situações em que existem descontos por quantidade ou qualquer outra alteração na estrutura de custos dos sistemas. Além disso, não são analisados os artigos de moda, tais como roupas e acessórios, e os bens perecíveis, tais como hortifrutigranjeiros.

O artigo divide-se em outras cinco partes: no item 2 há a descrição do problema; no 3, são apresentados os procedimentos selecionados na literatura; o item 4 apresenta o modelo de avaliação; o item 5 os ambientes em que os procedimentos são testados e o item 6 os resultados e as conclusões, além de sugestões para continuação do estudo.

\section{Descrição do Problema}

As empresas que operam no comércio varejista podem ter seu tipo de produção classificado como "estoque puro". Tais sistemas não executam atividades de manufatura em seus produtos, limitando-se a intermediar os bens entre seus fabricantes (ou atacadistas ) e seus clientes.

Desta forma, o tempo gasto para a execução de sua função (suprimento) é decorrente, basicamente, do tempo necessário à entrega dos bens pelos fornecedores 
(lead-time de compra). Além disso, o fornecedor pode exigir um tamanho mínimo de pedido, pedidos que envolvam múltiplos de uma certa quantidade ou valor, um período mínimo entre pedidos ou ainda uma periodicidade fixa para a sua colocação.

A demanda pelos seus produtos ocorre de forma quase contínua, em pequenos lotes ou mesmo em unidades, sendo que, em caso de falta do bem na "prateleira", o cliente, via de regra, dirige-se a outra empresa para adquirí-lo.

As decisões operacionais da empresa, neste caso, limitam-se a definir os instantes em que serão efetuados os pedidos e a quantidade dos pedidos de cada bem, sendo que o intervalo de tempo em que estas decisões são tomadas (período de replanejamento) costuma variar desde um dia até, normalmente, um mês.

Tais decisões devem levar em consideração os custos associados a estoques, os quais podem ser classificados em quatro grandes grupos: pedido (preparação e colocação de ordens, recepção e controle de qualidade dos bens), material (o bem em si), estocagem (oportunidade do capital empatado, armazenagem, seguro, deterioração, obsolescência, etc) e falta (nos casos que serão estudados, a perda de venda acarreta, além da perda da margem bruta de contribuição do produto, a perda de imagem frente aos clientes e o ônus de eventuais esforços para contornar a situação).

Os custos do sistema de gestão serão desconsiderados. Esta desconsideração não deve levar a grandes erros, pois há uma tendência à utilização de "terminais de pontos de venda" para atualização dos níveis de estoque e emissão de relatórios, fazendo com que os custos de apontamento sejam independentes da política adotada (uma política define uma lógica de funcionamento para o sistema, não especificando os parâmetros para este funcionamento, como fazem os procedimentos).

Não parece razoável supor que as empresas de varejo dos bens considerados neste trabalho possam adotar uma política de revisão contínua (apontamento e execução de pedidos continuamente ao longo do tempo), devido às políticas de atendimento de seus fornecedores (que não reprogramam suas entregas continuamente) ou aos altos custos que teria um tratamento especial pelos fornecedores. Com isto, para o tipo de empresa (e de bens) em questão, as políticas que melhor aderem à realidade são aquelas nas quais a empresa, periodicamente (a cada dia ou semana, por exemplo), verifica seu nível de estoque (I(t)) e, caso este se encontre abaixo de um certo referencial (s), (1) efetua um pedido de uma certa quantidade $(Q)$ ou (2) efetua um pedido que eleve o nível até um patamar desejado (Z). Uma terceira política, denominada (R,Z), define que o nível de reposição s deve ser igual ao nível Z (o que, na prática, faz com que, obrigatoriamente, um novo pedido seja feito a cada revisão). Por fim, pode ser adotada uma política baseada no cálculo de necessidades, na qual se faz, a intervalos regulares, um pedido referente à demanda projetada para o intervalo de tempo subseqüente ao lead-time (R,Q).

O objetivo da operação do sistema não se resume a minimizar custos pois a receita não constitui um valor fixo (o que também ocorreria no caso de atraso, se considerássemos o valor do dinheiro no tempo). Com isto, este objetivo pode ser expresso como sendo o de maximizar uma margem total (MT), dada por:

$\mathrm{H}$

MT=RU. $\quad(\operatorname{mín}(\mathrm{d}(\mathrm{t}), \mathrm{II}(\mathrm{t})))-\left(\mathrm{c}_{1} \cdot \mathrm{m}+\mathrm{c}_{5} \cdot \quad(\mathrm{d}(\mathrm{t})-\mathrm{I}(\mathrm{t}-1))^{+}+\right.$ 


$$
\left.{ }_{+\mathrm{c}_{3} \cdot \underset{\mathrm{t}=1}{\mathrm{t}=1}(\mathrm{I}(\mathrm{t}-1)-\mathrm{d}(\mathrm{t}))^{+}+\mathrm{c}_{\mathrm{t}=1} \mathrm{H}}^{\mathrm{H}} \mathrm{Q}(\mathrm{t})\right),
$$

$\mathrm{RU}$ = receita unitária de venda

$\mathrm{d}(\mathrm{t})=$ demanda do bem no período $\mathrm{t}$

II(t) = estoque no início do período, após a chegada do pedido

$\mathrm{c} 1=$ custo unitário de pedido

$\mathrm{m}$ = quantidade de pedidos realizados no horizonte $\mathrm{H}$

c5 = custo de falta adicional - para recuperar a imagem da empresa, por exemplo -

(c2, o custo unitário de falta, inclui a perda da margem de contribuição unitária)

$\mathrm{I}(\mathrm{t})$ = estoque final no período $\mathrm{t}$

c3 = custo unitário de estocagem

$\mathrm{c}_{4}=$ custo unitário de material

$\mathrm{Q}(\mathrm{t})=$ compra realizada no período $\mathrm{t}$

()$^{+}=$máximo entre zero e o valor entre parênteses

Para a consideração do valor do dinheiro no tempo, basta dividir o custo incorrido num certo dia t por $\left(1+j^{\prime}\right)$, onde j' indica a taxa de desconto por período.

O problema da empresa consiste, portanto, em definir os instantes e as quantidades a adquirir em função de uma demanda estimada, de modo a maximizar a diferença entre a receita e os custos totais resultantes da forma de operação num horizonte de tempo longo.

\section{Procedimentos Selecionados}

Diversos modelos foram concebidos para a solução do problema levantado nos itens anteriores, embora nenhum de uso geral. Pode-se classificá-los segundo as hipóteses utilizadas. A partir dos modelos foram desenvolvidos procedimentos. As hipóteses mais relevantes seriam as relativas à periodicidade da revisão (contínua ou não), ao comportamento da demanda (estática ou dinâmica), à certeza da demanda e do lead-time (determinísticos ou probabilísticos), à variabilidade do pedido (fixo ou não) e ao horizonte de planejamento (finito ou infinito).

O criador de cada sistema utilizou hipóteses para a construção de um modelo, admitiu uma política para ser utilizada ((s,Q), (s,Z), etc) e delineou uma série de passos para a definição dos parâmetros do modelo e para sua utilização efetiva. $\mathrm{O}$ termo "procedimento" será utilizado para identificar um método de administração do sistema.

Um procedimento pode ser utilizado mesmo numa situação na qual as hipóteses nas quais se baseia não são verificadas. Um conjunto de procedimentos baseados em hipóteses semelhantes constitui uma "família de procedimentos".

\section{A) Demanda determinística e estável, horizonte infinito e revisão contínua.}


O chamado "lote econômico de Wilson" (SILVER \& PETERSON, 1985) é o modelo de concepção e aplicação mais simples, embora apresente as hipóteses menos realistas. Aplicado à situação em estudo, na qual temos, sempre, revisão periódica, lead-time probabilístico, possibilidade de faltas (perda de venda), pedidos solicitados e não recebidos e demanda probabilística, o procedimento torna-se o seguinte:

\section{Procedimento A1}

(i) $\mathrm{s}=\mathrm{d}_{\mathrm{m}} \cdot \mathrm{L}_{\mathrm{m}}, \mathrm{s}=$ nível de reposição

$\mathrm{d}_{\mathrm{m}}=$ demanda média no período

$\mathrm{L}_{\mathrm{m}}=$ lead-time médio

(ii) em cada instante de revisão;

$$
\begin{aligned}
& \text { se } \mathrm{I}(\mathrm{t})+\text { QESP } \quad \mathrm{s}, \mathrm{Q}(\mathrm{t})=\left(2 \cdot \mathrm{c}_{1} \cdot \mathrm{d}_{\mathrm{m}} / \mathrm{c}_{3}\right)^{1 / 2} \\
& \text { se I }(\mathrm{t})+\text { QESP } \quad \mathrm{s}, \mathrm{Q}(\mathrm{t})=0, \\
& \mathrm{QESP}=\text { pedidos efetuados por chegar; } \mathrm{c}_{1}=\text { custo unitário de pedido } \\
& \mathrm{c} 3 \quad=\text { custo unitário de estocagem; } \mathrm{Q}(\mathrm{t})=\text { quantidade a ser pedida }
\end{aligned}
$$

Como a demanda é probabilística, pode haver períodos nos quais as unidades em estoque serão insuficientes, enquanto em outros sobrarão unidades em estoque após as vendas.

Como o "lote de Wilson" parte do princípio de que a revisão é contínua, o lote dimensionado pela fórmula anterior sugere um intervalo entre reposições igual a $\left(2 . c_{1} /\left(c_{3} \cdot d_{m}\right)\right)^{1 / 2}$ ("período econômico"), que, em função das relações entre os custos e a demanda, pode ser menor do que o período de revisão real do sistema. Caso isto ocorra, o modelo, sistematicamente, pedirá uma quantidade insuficiente para atender todo o período de revisão. Para proteger o modelo contra esta possibilidade, haverá (neste e nos demais procedimentos das famílias "A", "B" e "E") um limite mínimo para o lote de compra, igual à demanda projetada para o período de revisão (além do limite eventualmente imposto pelo fornecedor).

Além destas restrições, há a possibilidade de que o fornecedor exija que o lote seja múltiplo de um certo valor; neste caso, o lote pedido seria o menor múltiplo deste valor acima do lote sugerido pelo passo (ii). $\mathrm{O}$ fornecedor também pode exigir um intervalo mínimo entre dois pedidos consecutivos (novamente, há uma proteção para o caso de o passo (ii) sugerir um intervalo menor do que o imposto pelo fornecedor).

Por fim, deve-se definir o instante em que o lote solicitado ao fornecedor estará disponível para ser utilizado. O lead-time (que será gerado aleatoriamente, segundo uma distribuição uniforme, com valores mínimo e máximo), indicará uma disponibilidade, obrigatoriamente, não anterior à disponibilidade do pedido feito anteriormente, não havendo "cruzamento de ordens" (se o lead-time gerado for menor do que o intervalo até a disponibilidade de um pedido feito anteriormente, os dois pedidos estarão disponíveis no instante definido para o primeiro dos pedidos). 
Um modelo apresentado em LOVE (1979) sugere a utilização de um nível superior de referência (Z). Partindo-se do anterior e adaptando para as condições do estudo, temos:

\section{Procedimento A2}

(i) $\mathrm{s}=\mathrm{d}_{\mathrm{m}} \cdot \mathrm{L}_{\mathrm{m}}$

(ii) $\mathrm{Z}=\mathrm{s}+\left(2 \cdot \mathrm{c}_{1} \cdot \mathrm{d}_{\mathrm{m}} / \mathrm{c}_{3}\right)^{1 / 2}$

(iii) em cada instante de revisão;

$$
\begin{array}{ll}
\text { se } I(t)+Q_{E S P} & s, Q(t)=Z-I(t) \\
\text { se } I(t)+Q_{E S P} & s, Q(t)=0
\end{array}
$$

\section{B) Demanda probabilística e estável, horizonte infinito, revisão contínua e lead- time determinístico.}

O modelo a seguir, mais realista (Hadley e Whitin (1963, apud LOVE (1979)), considera a possibilidade de perda de venda e demanda probabilística, sendo sensivelmente mais complexo para a definição de seus parâmetros, como apresentado abaixo:

\section{Procedimento B1}

(i) processo iterativo para encontrar s e $\mathrm{Q}\left(\mathrm{com} \mathrm{b}_{\mathrm{m}}(\mathrm{s})\right.$, a falta média no período, igualada a zero na primeira iteração)

1ㅇ) $\quad \mathrm{Q}=\left(2 \cdot \mathrm{d}_{\mathrm{m}} \cdot\left(\mathrm{c}_{1}+\mathrm{c}_{2} \cdot \mathrm{b}_{\mathrm{m}}(\mathrm{s})\right) / \mathrm{c}_{3}\right)^{1 / 2}$

$\mathrm{C}_{2}=$ custo unitário de falta

20) $\quad \mathrm{F}_{\mathrm{L}}(\mathrm{s})=\left(\mathrm{c}_{2} \cdot \mathrm{d}_{\mathrm{Lm}} / \mathrm{Q}\right) /\left(\mathrm{c}_{2} \cdot \mathrm{d}_{\mathrm{Lm}} / \mathrm{Q}+\mathrm{c}_{3}\right)$,

$\mathrm{F}_{\mathrm{L}}(\mathrm{s})$ sendo a função de distribuição acumulada da

normal no ponto s e $\mathrm{d}_{\mathrm{Lm}}$ a demanda média no lead-time

30) $\mathrm{s}=\mathrm{d}_{\mathrm{Lm}}+\mathrm{z}\left(\mathrm{F}_{\mathrm{L}}(\mathrm{s})\right) . \mathrm{L}$,

$\mathrm{z}$ sendo a abcissa da normal padrão e

$\mathrm{L}$ o desvio-padrão da demanda no lead-time

40) $\quad b_{m}(s)=L \cdot I\left(\left(s-d_{L m}\right) / L\right)$,

$\mathrm{I}(\mathrm{x})$ sendo a integral de perda da normal padrão

(ii) continuamente (em cada instante de revisão, no presente estudo):

se I $(\mathrm{t})+$ QESP $\quad \mathrm{s}, \mathrm{Q}(\mathrm{t})$ conforme passos acima

se $I(t)+$ QESP $\quad s, Q(t)=0$

O modelo acima, que parte de uma simplificação para definir os parâmetros (considera o intervalo entre pedidos igual $\mathrm{d}_{\mathrm{m}} / \mathrm{Q}$ e não a $\left(\mathrm{d}_{\mathrm{m}}-\mathrm{b}_{\mathrm{m}}(\mathrm{s}) / \mathrm{L}\right) / \mathrm{Q}$, resultaria no seguinte custo total por período (sem considerar o custo de material):

$\mathrm{CTP}=\mathrm{c}_{1} \cdot \mathrm{d}_{\mathrm{m}} / \mathrm{Q}+\mathrm{c}_{2} \cdot\left(\mathrm{Q} / 2+\mathrm{s}-\mathrm{d}_{\mathrm{m}} \cdot \mathrm{L}+\mathrm{b}_{\mathrm{m}}(\mathrm{s})\right)+\mathrm{c}_{3} \cdot \mathrm{b}_{\mathrm{m}}(\mathrm{s}) \cdot \mathrm{d}_{\mathrm{m}} / \mathrm{Q}$

Outro procedimento possível (Hadley e Whitin (1963, apud LOVE (1979)) utiliza a política de estoque base (Z) o qual, adaptado para a situação, resulta em: 


\section{Procedimento B2}

(i) $\mathrm{F}_{\mathrm{L}}(\mathrm{Z})=\left(\mathrm{c}_{2} \cdot \mathrm{d}_{\mathrm{m}} / \mathrm{Q}_{\mathrm{m}}\right) /\left(\mathrm{c}_{2} \cdot \mathrm{d}_{\mathrm{m}} / \mathrm{Q}_{\mathrm{m}}+\mathrm{c}_{3}\right)$, onde $\mathrm{Q}_{\mathrm{m}}$ é o número médio de unidades demandadas por incidência de demanda (valor estimado para o bem em questão, igualado a 1 nos testes realizados)

(ii) $\mathrm{Z}=\mathrm{d}_{\mathrm{m}} \cdot \mathrm{L}_{\mathrm{m}}+\mathrm{z}\left(\mathrm{F}_{\mathrm{L}}(\mathrm{Z})\right) \cdot \mathrm{L}, \quad \mathrm{L}_{\mathrm{m}}$ sendo o lead-time médio

(iii) em cada instante de revisão:

$$
\begin{array}{ll}
\text { se } I(t)+Q_{E S P} & Z, Q(t)=Z-I(t) \\
\text { se I }(t)+Q_{E S P} & Z, Q(t)=0
\end{array}
$$

Outros modelos, que utilizam a política de quantidade fixa (portanto, o último passo igual ao (ii) de B1), podem ser apresentados:

Procedimento B3 (Hadley e Whitin (1963, apud SILVER \& PETERSON (1985))

(i) Q obtido por meio de alguma fórmula (por exemplo, pelo lote de Wilson)

(ii) $\mathrm{G}_{\mathrm{U}}(\mathrm{k})=\mathrm{Q} / \mathrm{L} \cdot \mathrm{c}_{3}\left(\mathrm{c}_{2}+\mathrm{c}_{3}\right)$, onde $\mathrm{G}_{\mathrm{u}}(\mathrm{k})$ é a função de expectativa parcial

demanda

$$
\text { k o fator de segurança para atendimento da }
$$

(iii) $\mathrm{s}=\mathrm{k} \cdot \mathrm{L}+\mathrm{d}_{\mathrm{Lm}}$

(iv) em cada instante de revisão:

se $\mathrm{I}(\mathrm{t})+$ QESP $\quad \mathrm{s}, \mathrm{Q}(\mathrm{t})=\mathrm{Q}$, conforme passos acima

se $I(t)+$ QESP $\quad s, Q(t)=0$

\section{Procedimento B4 (HAX \& CANDEA, 1984)}

(i) $\mathrm{Q}=\left(2 \cdot \mathrm{d}_{\mathrm{m}} \cdot\left(\mathrm{c}_{1}+\mathrm{c}_{2} \cdot \mathrm{G}_{\mathrm{u}}(\mathrm{k})\right) / \mathrm{c}_{3}\right)^{1 / 2}, \quad$ processo iterativo com $\mathrm{G}_{\mathrm{u}}(\mathrm{k})$ igual a zero na 1 a iteração

(ii) $\mathrm{P}(\mathrm{k})=\mathrm{Q} \cdot \mathrm{c}_{3} /\left(\mathrm{d}_{\mathrm{m}} \cdot \mathrm{c}_{2}\right)$, onde $\mathrm{P}(\mathrm{k})$ é a função de probabilidade acumulada para $\mathrm{k}$

(iii) $\mathrm{s}=\mathrm{k} \cdot \mathrm{L}+\mathrm{d}_{\mathrm{Lm}}$

(iv) em cada instante de revisão:

se $\mathrm{I}(\mathrm{t})+\mathrm{QESP}_{\mathrm{ESP}} \mathrm{s}, \mathrm{Q}(\mathrm{t})=\mathrm{Q}$, conforme passos acima

se $I(t)+Q_{E S P} \quad s, Q(t)=0$

Procedimento B5 (MABIN, 1988)

(i) $\mathrm{Q}=.+\left((\cdot)^{2}+\mathrm{EOQ}^{2}\right)^{1 / 2}$, onde EOQ $($ Wilson $)=\left(2 \cdot \mathrm{c}_{1} \cdot \mathrm{d}_{\mathrm{m}} / \mathrm{c}_{3}\right)^{1 / 2} \mathrm{e}$ conforme a tabela abaixo:

\begin{tabular}{|c|c|c|c|c|c|c|c|c|}
\hline $\mathrm{c}_{2} /\left(\mathrm{c}_{2}+\mathrm{c} 3\right)$ & 80,0 & 90,0 & 92,5 & 95,0 & 97,5 & 98,0 & 99,0 & 99,5 \\
\hline & 0,7125 & 0,5585 & 0,5185 & 0,4740 & 0,4180 & 0,4045 & 0,3687 & 0,3390 \\
\hline
\end{tabular}


(ii) $\mathrm{G}_{\mathrm{u}}(\mathrm{k})=\mathrm{Q} / \mathrm{L} \cdot \mathrm{c}_{3}\left(\mathrm{c}_{2}+\mathrm{c}_{3}\right)$

(iii) $\mathrm{s}=\mathrm{k} \cdot \mathrm{L}+\mathrm{d}_{\mathrm{Lm}}$

(iv) em cada instante de revisão:

se $\mathrm{I}(\mathrm{t})+\mathrm{QESP} \quad \mathrm{s}, \mathrm{Q}(\mathrm{t})=\mathrm{Q}$, conforme passos acima

se $I(t)+$ QESP $\quad s, Q(t)=0$

Este procedimento também é heurístico, considerando a possibilidade de atendimento com atraso (não viável nas situações do presente estudo).

Procedimento B6 (DAS, 1985)

(i) $\mathrm{g}=\mathrm{c}_{2} \cdot \mathrm{d}_{\mathrm{m}} /\left(2 \cdot \mathrm{c}_{3} \cdot\right)$ $\mathrm{v}=\mathrm{c}_{1} /\left(\mathrm{c}_{2} \cdot\right)$

(ii) $\mathrm{P}=\left(\mathrm{b}+\left(\mathrm{b}^{2}+4 \cdot(\mathrm{g}-\mathrm{a}) \cdot(\mathrm{v}-\mathrm{c})\right)^{1 / 2}\right) /(2 \cdot(\mathrm{g}-\mathrm{a}))$ onde: $\mathrm{a}=0,79838 ; \mathrm{b}=0,39694 ; \mathrm{c}=-0,0000044$

(iii) $\mathrm{Q}=$ 2.g.P.

(iv) $\mathrm{s}=\mathrm{k} \cdot \mathrm{L}+\mathrm{d}_{\mathrm{Lm}}$, onde $\mathrm{P}(\mathrm{k})=\mathrm{c}_{2} /\left(\mathrm{c}_{2}+\mathrm{c}_{3}\right)$

(v) em cada instante de revisão:

se $\mathrm{I}(\mathrm{t})+\mathrm{QESP} \quad \mathrm{s}, \mathrm{Q}(\mathrm{t})=\mathrm{Q}$, conforme passos acima

se $I(t)+Q_{E S P} \quad s, Q(t)=0$

DAS (1985) compara sua aproximação quadrática da probabilidade de faltas à aproximação exponencial feita por Byrkett (1981, apud DAS (1985)) chegando à conclusão de que a sua produz melhores resultados.

\section{C) Demanda probabilística e estável, horizonte infinito e revisão periódica.}

Os resultados apresentados por Scarf (1960) e Iglehart (1963) apud PORTEUS (1985) demonstram que, para situações em que há possibilidade de atraso, as políticas $(\mathrm{s}, \mathrm{Z})$ produzem os melhores resultados. Entretanto, nada foi encontrado que pudesse extrapolar a afirmação para a situação de perda de venda. Esta política é a sugerida para tratar os problemas discutidos neste trabalho. Os procedimentos propostos seriam:

Procedimento C1 (Hadley \& Whitin, 1963 apud LOVE (1979))

Love sugere a análise de um parâmetro para se optar entre dois procedimentos heurísticos. Caso o valor de $\mathrm{c}_{1}$ seja maior do que $1,125 \cdot \mathrm{d}_{\mathrm{m}} \cdot \mathrm{c}_{2} \cdot \mathrm{c}_{3} /\left(\mathrm{c}_{2}+\mathrm{c}_{3}\right)$, deve-se optar por uma política (s,Z), cujos passos seriam:

(i) $\mathrm{sD}_{\mathrm{D}}=\left(2 \cdot \mathrm{d}_{\mathrm{m}} \cdot \mathrm{c}_{1} / \mathrm{c}_{3}\right)^{1 / 2} \cdot\left(\mathrm{c}^{2} /\left(\left(\mathrm{c}_{3}+\mathrm{c}_{2}\right) \cdot \mathrm{c}_{2}\right)\right)^{1 / 2}$

(ii) $\mathrm{QD}_{\mathrm{D}}=\left(2 \cdot \mathrm{d}_{\mathrm{m}} \cdot \mathrm{c}_{1} / \mathrm{c}_{3}\right)^{1 / 2} \cdot\left(\left(\mathrm{c}_{2}+\mathrm{c}_{3}\right) / \mathrm{c}_{2}\right)^{1 / 2}$

(iii) $\mathrm{s}=\mathrm{d}_{\mathrm{m}} \cdot \mathrm{T} / 2+\mathrm{d}_{\mathrm{m}} \cdot(\mathrm{T}+\mathrm{L})+\mathrm{s}_{\mathrm{D}}$ 
(iv) $\mathrm{Z}=\mathrm{Q}_{\mathrm{D}}+\mathrm{s}-\mathrm{d}_{\mathrm{m}} \cdot \mathrm{T} / 2$

(v) em cada instante de revisão:

$$
\begin{array}{ll}
\text { se } I(t)+\text { QESP } & \text { s, Q }(t)=Z-I(t) \\
\text { se } I(t)+\text { QESP } & s, Q(t)=0
\end{array}
$$

Caso contrário, c1 pode ser desprezado. o que significa que a política alternativa seria pedir em todos os períodos o suficiente para atingir o nível Z (política $(T, Z)$ ), conforme a seguir:

(i) $\mathrm{F}_{\mathrm{T}+\mathrm{L}}(\mathrm{Z})=\mathrm{C}_{2} /\left(\mathrm{c}_{2}+\mathrm{C}_{3}\right)$

(ii) $\mathrm{Z}=\mathrm{d}_{\mathrm{m}} \cdot(\mathrm{T}+\mathrm{L})+\mathrm{z} \cdot \mathrm{T}+\mathrm{L}, \mathrm{F}_{\mathrm{T}}+\mathrm{L}(\mathrm{Z})$ é a função de distribuição acumulada da demanda no intervalo $\mathrm{T}+\mathrm{L}$, $\mathrm{z}$ o valor da abcissa no ponto correspondente e $\mathrm{T}+\mathrm{L}$ o desvio-padrão da demanda

(iii) em cada instante de revisão:

$$
\begin{array}{ll}
\text { se } I(t)+\text { QESP } & Z, Q(t)=Z-I(t) \\
\text { se } I(t)+\text { QESP } & Z, Q(t)=0
\end{array}
$$

Caso o intervalo entre pedidos imposto pelo fornecedor (M), seja maior do que o período entre revisões inicialmente desejado pela empresa (T), este intervalo deve substituir o período "T" (colocação válida também para o procedimento seguinte, no qual o período entre revisões é denotado por R).

Outro procedimento heurístico (que considera atraso na entrega), desenvolvido por Ehrardt (1979, apud SILVER \& PETERSON (1985)) seria:

Procedimento C2 (Ehrardt, 1979)

(i) $\mathrm{x}_{\mathrm{R}}=\mathrm{d}_{\mathrm{m}} \cdot \mathrm{R}$

$$
\begin{aligned}
\mathrm{x}_{\mathrm{R}+\mathrm{L}} & =\mathrm{d}_{\mathrm{m}} \cdot(\mathrm{R}+\mathrm{L}) \\
\mathrm{R}+\mathrm{L} & =\mathrm{L} \cdot((\mathrm{R}+\mathrm{L}) / \mathrm{L})^{1 / 2}
\end{aligned}
$$

(ii) $\mathrm{Q}_{\mathrm{p}}=1,30 \cdot \mathrm{x}_{\mathrm{R}} \mathrm{R}^{0,494} \cdot\left(\mathrm{c}_{1} / \mathrm{c}_{3}\right)^{0,506} \cdot\left(1+\mathrm{L}^{2 / \mathrm{x}_{\mathrm{R}}}\right)^{0,116}$

(iii) $\mathrm{z}=\left(\mathrm{Q}_{\mathrm{p}} \cdot \mathrm{c}_{3} /\left(\mathrm{R}+\mathrm{L} \cdot \mathrm{c}_{2}\right)\right)^{1 / 2}$

(iv) $\mathrm{s}_{\mathrm{p}}=0,973 \cdot \mathrm{x} \mathrm{R}+\mathrm{L}+\mathrm{R}+\mathrm{L} \cdot(0,183 / \mathrm{z}+1,063-2,192 \cdot \mathrm{z})$

(v) se $Q_{p} / x_{R} \quad 1,5$ então $\quad s=s_{p}$

$$
\begin{array}{ll}
\mathrm{Z}=\mathrm{s}_{\mathrm{p}}+\mathrm{Q}_{\mathrm{p}} \\
\text { senão } & \mathrm{Z}_{0}=\mathrm{x}_{\mathrm{R}+\mathrm{L}}+\mathrm{k} . \mathrm{R}+\mathrm{L}, \mathrm{P}(\mathrm{k})=\mathrm{c}_{2} /\left(\mathrm{c}_{2}+\mathrm{c}_{3}\right)
\end{array}
$$$$
\mathrm{s}=\min \left(\mathrm{s}_{\mathrm{p}}, \mathrm{Z}_{0}\right)
$$

(vi) em cada instante de revisão:

$$
\mathrm{Z}=\min \left(\mathrm{s}_{\mathrm{p}}+\mathrm{Q}_{\mathrm{p}}, \mathrm{Z}_{0}\right)
$$

se $\mathrm{I}(\mathrm{t})+$ QESP $\quad \mathrm{s}, \mathrm{Q}(\mathrm{t})=\mathrm{Z}-\mathrm{I}(\mathrm{t})$

se $I(t)+$ QESP $\quad s, Q(t)=0$ 
O procedimento seguinte, também heurístico, com erros inferiores a 1\% com relação ao analítico, segundo NADDOR (1975), seria adequado a uma política (R,Z), no qual se procura definir economicamente um período ótimo entre reposições:

Procedimento C3 (NADDOR, 1975)

(i) $\mathrm{R}=$ inteiro mais próximo de $\left(2 . \mathrm{c}_{1} /\left(\mathrm{c}_{3} \cdot \mathrm{A} \cdot \mathrm{d}_{\mathrm{m}}\right)\right)^{1 / 2}$, onde $\mathrm{A}=\mathrm{c}_{2} /\left(\mathrm{c}_{2}+\mathrm{c}_{3}\right)$

(ii) $\mathrm{Z}=\mathrm{y}_{\mathrm{m}}+\mathrm{k} \cdot \mathrm{y}$,

$$
\begin{aligned}
& \mathrm{y}_{\mathrm{m}}=\left(\mathrm{L}_{\mathrm{m}}+\mathrm{R} / 2\right) \cdot \mathrm{d}_{\mathrm{m}} \\
& \mathrm{y}=\left(\left(\mathrm{L}_{\mathrm{m}}+\mathrm{R} / 3\right) .{ }^{2}+\left(\mathrm{R} \cdot \mathrm{d}_{\mathrm{m}}\right)^{2} / 12+\mathrm{u}^{2}\left(1-\mathrm{P}_{0} \mathrm{R}\right) / 6\right)^{1 / 2} \text {, } \\
& \text { se I }(\mathrm{t})+\text { QESP } \quad \mathrm{Z}, \mathrm{Q}(\mathrm{t})=\mathrm{Z}-\mathrm{I}(\mathrm{t}) \\
& \text { se } I(t)+Q_{E S P} \quad Z, Q(t)=0
\end{aligned}
$$$$
\text { é o desvio-padrão da demanda por intervalo de tempo, }
$$

u é a unidade básica de compra (por exemplo, uma dúzia) e

$\mathrm{P}_{0}$ a probabilidade de que a demanda no período seja igual

a zero (nos testes realizados, esta probabilidade é nula).

Neste procedimento e no seguinte, caso o intervalo entre pedidos imposto pelo fornecedor seja maior do que o período econômico ótimo, o valor de $\mathrm{M}$ deve substituir $\mathrm{R}$ ( $\mathrm{t}_{0}$, no procedimento seguinte).

O último modelo classificado como de revisão periódica, mas que opta por elevar o nível de estoque a um valor $\mathrm{Z}$, sempre que I(t) s, seria:

Procedimento C4 (NADDOR, 1975)

(i) $\mathrm{Q}_{0}=\left(2 \cdot \mathrm{d}_{\mathrm{m}} \cdot \mathrm{c}_{1} /\left(\mathrm{c}_{3} \cdot \mathrm{A}\right)\right)^{1 / 2}$ (múltiplo de u)

(ii) $\mathrm{t}_{0}=\left(2 . \mathrm{c}_{1} /\left(\mathrm{c}_{3} \cdot \mathrm{A} \cdot \mathrm{d}_{\mathrm{m}}\right)\right)^{1 / 2}$ (múltiplo de R)

(iii) $\mathrm{Z}=\mathrm{y}_{\mathrm{m}}\left(\mathrm{t}_{0}\right)+\mathrm{k} . \quad \mathrm{y}\left(\mathrm{t}_{0}\right)$

(iv) $\mathrm{s}=\mathrm{Z}-\mathrm{Q}_{0}$

(v) em cada instante de revisão:

$$
\begin{array}{ll}
\text { se } I(t)+Q_{E S P} & s, Q(t)=Z-I(t) \\
\text { se } I(t)+Q_{E S P} & s, Q(t)=0
\end{array}
$$

Este procedimento também é apresentado como uma boa aproximação do modelo analítico (diferenças encontradas inferiores a 5\%).

PORTEUS (1985) executou um estudo semelhante ao proposto neste trabalho, limitando-se, porém, a verificar o comportamento de procedimentos heurísticos desenvolvidos para situações em que há possibilidade de atendimento com atraso, utilizados em políticas (s,Z). Sua intenção foi a de apresentar procedimentos com execução simples, para implementação prática. Para tanto, testou 17 procedimentos, comparando-os ao custo médio do procedimento analítico ótimo. Tais procedimentos 
não serão utilizados neste trabalho, pois outros semelhantes, que trazem a hipótese de atraso, já estão representados no estudo.

Para demandas com distribuição exponencial, que não serão simuladas neste trabalho, SNYDER (1974) apresenta duas formas de se obter s (o valor de Z é igual a

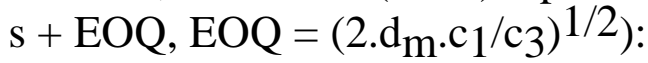

a) $\exp \left(-\mathrm{s} / \mathrm{d}_{\mathrm{m}}\right)=\mathrm{c}_{3} /\left(\mathrm{c}_{2}+\mathrm{c}_{3}\right) \cdot\left(\mathrm{EOQ} /\left(\mathrm{d}_{\mathrm{m}}+1\right)\right)$

b) $\exp \left(-\mathrm{s} / \mathrm{d}_{\mathrm{m}}\right)=\mathrm{c}_{3} / \mathrm{c}_{2} \cdot \mathrm{EOQ}$

\section{D) Demanda variável}

Modelos mais gerais, que permitem a consideração de demandas variáveis no tempo, têm sido objeto de estudos recentes, exceção feita aos modelos que utilizam técnicas de programação dinâmica para sua resolução (Wagner-Whitin, Silver-Meal e Fordyce-Webster), os quais não consideram demandas probabilísticas e possibilidade de faltas.

SILVER \& PETERSON (1985) apresentam um procedimento para produtos no fim da vida útil, devendo ser feito um último pedido, que atenderia toda a demanda com uma certa probabilidade. Brosseau (1982, apud SILVER \& PETERSON (1985), propõe o seguinte procedimento, para uma demanda linearmente decrescente e determinística $(\mathrm{d}(\mathrm{t})=\mathrm{a}-\mathrm{b} . \mathrm{t})$ :

(i) $\mathrm{M}=\mathrm{c}_{1} \cdot \mathrm{b}^{2} /\left(\mathrm{c}_{3} \cdot \mathrm{a}^{3}\right)$

(ii) se $\mathrm{M}>0,075$, então utiliza-se o lote de Wilson senão $\mathrm{Q}=\mathrm{a}^{2} /(2 . \mathrm{b})$ (último pedido)

RITCHIE (1984), RITCHIE \& TSADO (1985), MURDESHWAR (1988) e DAVE (1989a), prosseguindo estudos de Donaldson (1977, apud DAVE (1989a)), tratam de problemas com demandas linearmente crescentes e determinísticas, para horizontes finitos. Os procedimentos sugerem a divisão do horizonte em n períodos diferentes, encontrando-se a seguir as quantidades dos pedidos em cada instante (os modelos consideram lead-time igual a zero). O número ótimo de divisões é encontrado fazendo-o variar (a partir de 1) e analisando uma certa contribuição marginal para a redução do custo total (quando a contribuição se torna negativa, pára-se o processo).

Entretanto, Ritchie e Tsado sugerem que o lote de Wilson é uma ótima aproximação para este problema, que, aliás, é sensivelmente mais simples do que o estudado neste trabalho.

DAVE (1989b) apresenta um procedimento heurístico, que produz resultados muito próximos aos do analítico desenvolvido por ele, que permite a possibilidade de atrasos, conforme indicado abaixo:

Procedimento D1 (DAVE, 1989b)

(i) $\mathrm{M}=\mathrm{c}_{1} / \mathrm{c}_{3}$

$\mathrm{N}=\mathrm{C}_{3} / \mathrm{c}_{2}$

(ii) $\mathrm{R}(\mathrm{r})=((1+\mathrm{N}) \cdot \mathrm{M} /((\mathrm{a} / 2+(2+\mathrm{N}) \cdot \mathrm{b} \cdot \mathrm{R}(\mathrm{r}-1)) / 3 \cdot(1+\mathrm{N})))^{1 / 2}$ 
$\operatorname{com} \mathrm{R}(\mathrm{r}=0)=0$ na primeira iteração. Caso $\mathrm{R}$ resulte em valor menor do que 1 , assume-se $\mathrm{R}=1$. Caso $\mathrm{R}$ seja fracionário, aproxima-se para um valor inteiro. Finalmente, caso M (o intervalo mínimo imposto pelo fornecedor) seja maior do que R, adota-se $\mathrm{M}$ como o intervalo entre revisões

(iii) $\mathrm{Q}=\mathrm{a} \cdot \mathrm{R}+\mathrm{b} \cdot \mathrm{R}^{2} / 2$

(iv) $\mathrm{s}=\mathrm{a} \cdot \mathrm{L}_{\mathrm{m}}+\mathrm{b} \cdot \mathrm{L}_{\mathrm{m}}{ }^{2} / 2$ (extrapolando-se o procedimento de DAVE, para o qual não havia lead-time)

(v) em cada instante de revisão:

$$
\begin{array}{ll}
\text { se } I(t)+Q_{E S P} & s, Q(t)=Q, \text { conforme passos acima } \\
\text { se I }(t)+\text { QESP } & s, Q(t)=0
\end{array}
$$

Caso não seja permitido atraso no atendimento, o procedimento simplifica-se para o proposto por Silver (1979, apud AMRANI \& RAND (1990)):

Procedimento D2 (Silver, 1979)

(i) $\mathrm{M}=\mathrm{c}_{1} / \mathrm{c}_{3}$

(ii) $\mathrm{R}(\mathrm{r})=(2 \cdot \mathrm{M} /(\mathrm{a}+(4 / 3) \cdot \mathrm{b} \cdot \mathrm{R}(\mathrm{r}-1)))^{1 / 2}, \mathrm{R}(\mathrm{r}=0)=0$

(iii) $\mathrm{Q}=\mathrm{a} \cdot \mathrm{R}+\mathrm{b} \cdot \mathrm{R}^{2} / 2$

(iv) $\mathrm{s}=\mathrm{a} \cdot \mathrm{L}_{\mathrm{m}}+\mathrm{b} \cdot \mathrm{L}_{\mathrm{m}}{ }^{2} / 2$

(v) em cada instante de revisão:

se I $(\mathrm{t})+$ QESP $\quad \mathrm{s}, \mathrm{Q}(\mathrm{t})=\mathrm{Q}$, conforme passos acima

se $I(t)+$ QESP $\quad s, Q(t)=0$

AMRANI \& RAND (1990) sugerem o uso de quatro técnicas heurísticas (bastante semelhantes entre si) para se encontrar o período de duração do pedido (para o problema com atraso), uma delas a proposta por Silver, exposta acima, e as demais colocadas a seguir. Sugere que, a partir da solução proposta por cada uma, escolhe-se a que gerar o menor custo (destas quatro, apenas a de Silver será avaliada). A técnica de TSADO (1985) troca o valor "4/3" da equação do passo (ii) pelo valor "1/3", enquanto a de Goyal e Gommes (1982, apud AMRANI \& RAND (1990)) troca o mesmo valor por "2/3" e a de RITCHIE (1984) por "0,86".

Finalmente, FRIEDMAN (1981) apresenta propostas para problemas com demanda e custo de estocagem exponenciais (os quais não serão tratados neste trabalho) e HENERY (1990) apresenta soluções para problemas com demandas senoidais, "dente-de-serra" e com mudança instantânea de patamar, sendo, entretanto, úteis apenas para um número definido e pequeno (até 3) de pedidos por ciclo (situação bastante diferente da que será considerada neste estudo, no qual os pedidos terão periodicidade da ordem de semanas e o ciclo será anual).

E) Demanda probabilística e estável, horizonte infinito, revisão contínua e leadtime probabilístico 
FRIEDMAN (1988) propõe uma fórmula para o cálculo do lote que considera a distribuição de probabilidades do lead-time de compra, como segue:

Procedimento E1 (FRIEDMAN, 1988)

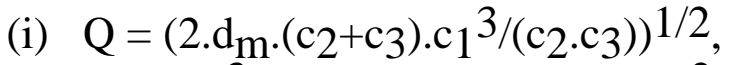
$\mathrm{c}_{1}{ }^{3}=\mathrm{c}_{1}+\mathrm{d}_{\mathrm{m}} \cdot\left(\mathrm{c}_{2}+\mathrm{c}_{3}\right) \cdot \mathrm{LT}^{2 / 2}$,

LT sendo o desvio-padrão do lead-time, que para

distribuição uniforme equivale a $\left(\left(\mathrm{LT}_{\text {máx }}-\mathrm{LT}_{\text {mín }}\right)^{2 / 12}\right)^{1 / 2}$

Para se encontrar s, será utilizada a seguinte abordagem (não original):

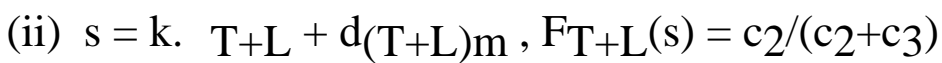

(iii) em cada instante de revisão:

se $\mathrm{I}(\mathrm{t})+\mathrm{QESP} \quad \mathrm{s}, \mathrm{Q}(\mathrm{t})=\mathrm{Q}$, conforme passos acima

se $I(t)+$ QESP $\quad s, Q(t)=0$

SPHICAS \& NASRI (1984), continuando estudo de Liberatore (1979, apud SPHICAS \& NASRI (1984)), propõem as seguintes fórmulas para se encontrar Q,

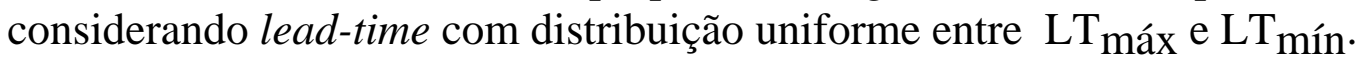

Procedimento E2 (SPHICAS \& NASRI, 1984)

(i) $\mathrm{Q}=\left(12 \cdot \mathrm{d}_{\mathrm{m} \cdot \mathrm{c} 1} \cdot\left(\mathrm{LT}_{\mathrm{máx}}-\mathrm{LT}_{\mathrm{mín}}\right) /\left(\mathrm{c}_{2}+\mathrm{C}_{3}\right)\right)^{1 / 3}$,

$$
\begin{aligned}
& \text { se 2.c } 1 /\left(\mathrm{d}_{\mathrm{m}} \cdot\left(\mathrm{c}_{2}+\mathrm{c}_{3}\right)\right) \quad\left(4 \cdot\left(\mathrm{c}_{3} / \mathrm{c}_{2}\right)^{3} /\left(3 \cdot\left(1+\left(\mathrm{c}_{3} / \mathrm{c}_{2}\right)^{3}\right)\right)\right) \cdot\left(\mathrm{LT}_{\mathrm{máx}}-\mathrm{LT}_{\mathrm{mín}}\right)^{2} \\
& \mathrm{Q}=\left(\left(2 \cdot \mathrm{d}_{\mathrm{m}} \cdot \mathrm{c}_{1} \cdot\left(\mathrm{c}_{2}+\mathrm{c}_{3}\right)+\mathrm{LT}^{2} \cdot \mathrm{d}_{\mathrm{m}^{2}} \cdot\left(\mathrm{c}_{2}+\mathrm{c}_{3}\right)^{2}\right) /\left(\mathrm{c}_{2} \cdot \mathrm{c}_{3}\right)\right)^{1 / 2} \\
& \text { se 2.c } 1 /\left(\mathrm{d}_{\mathrm{m}} \cdot\left(\mathrm{c}_{2}+\mathrm{c}_{3}\right)\right) \quad\left(\left(3-\mathrm{c}_{3} / \mathrm{c}_{2}\right) /\left(12 \cdot\left(\mathrm{c}_{3} / \mathrm{c}_{2}\right)\right)\right) \cdot\left(\mathrm{LT}_{\operatorname{máx}}-\mathrm{LT}_{\mathrm{mín}}\right)^{2} \\
& \mathrm{Q}=\mathrm{q} \cdot \mathrm{d}_{\mathrm{m}} \text {, onde: } \\
& \mathrm{q}^{2}-(2 / 3) \cdot\left(2 \cdot\left(\mathrm{LT}_{\operatorname{máx}}-\mathrm{LT}_{\mathrm{mín}}\right) /(1+\mathrm{m})\right)^{1 / 2} \cdot \mathrm{q}^{3 / 2}=\mathrm{c}_{1} \cdot(1+\mathrm{m}) \\
& \mathrm{m}=\operatorname{máx}\left(\mathrm{c}_{2} / \mathrm{c}_{3}, \mathrm{c}_{3} / \mathrm{c}_{2}\right),
\end{aligned}
$$

se 2. $\mathrm{c}_{1} /\left(\mathrm{d}_{\mathrm{m}} \cdot\left(\mathrm{c}_{2}+\mathrm{C}_{3}\right)\right)$ entre os valores acima

Novamente, para se encontrar s, será utilizada a fórmula exposta em E1:

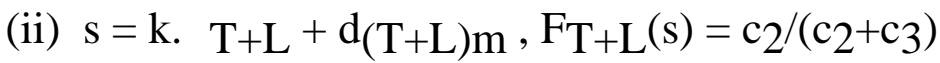

(iii) em cada instante de revisão:

$$
\begin{array}{ll}
\text { se } \mathrm{I}(\mathrm{t})+\mathrm{QESP} & \mathrm{s}, \mathrm{Q}(\mathrm{t})=\mathrm{Q} \text {, conforme passos acima } \\
\text { se } \mathrm{I}(\mathrm{t})+\mathrm{QESP} & \mathrm{s}, \mathrm{Q}(\mathrm{t})=0
\end{array}
$$

\section{F) Modelos derivados do cálculo de necessidades}

A última família de procedimentos a ser considerada no estudo consiste numa simplificação do cálculo de necessidades (materials requirements planning). Trata-se de uma simplificação particularmente pela inexistência de componentes de um produto principal, não havendo a chamada "demanda derivada", para a qual o cálculo de necessidades foi desenvolvido. 
Deste método será aproveitada a idéia de, simplesmente, defasar no tempo as necessidades de cada período ou a composição de algumas destas necessidades (para a formação de um lote de compra), para considerar a existência de lead-times para aquisição dos bens, além de descontar estoques já disponíveis das necessidades dos primeiros períodos.

Para a formação de pedidos de compra, poder-se-ia utilizar o algoritmo de Wagner e Whitin (1958, apud HAX \& CANDEA (1983)) ou a técnica substituta, também otimizante, desenvolvida por FORDYCE \& WEBSTER (1984). Entretanto, dadas as características dos problemas que são analisados neste estudo, como a constância dos custos de pedido, material e estocagem, além da pequena variação da demanda no curto prazo (exceto, talvez, na hipótese de sazonalidade em que há um pico de demanda num certo intervalo de tempo), técnicas heurísticas simples podem ser utilizadas. Por exemplo, caso, num certo momento, o custo de estocagem de toda a demanda de uma semana seja alto o suficiente para não justificar a compra da demanda de duas semanas num único lote (indicando que a compra deverá ser suficiente apenas para uma semana), esta lógica não deverá ser alterada, dadas as características dos problemas expostas acima.

Com isto, uma técnica heurística simples será utilizada para a formação dos lotes, o "método do período fixo", que combina a demanda de um certo número de períodos, sem alteração deste ao longo do tempo.

Além disto, como, na realidade, tanto a demanda como o lead-time são probabilísticos, serão adaptados meios para prover o sistema de segurança com relação a estas duas incertezas (conforme sugestão apresentada em HAX \& CANDEA (1984)) como apresentado a seguir. $\mathrm{O}$ primeiro dos procedimentos não considera as incertezas, o segundo apenas a incerteza da demanda, o terceiro apenas a incerteza do lead-time e o quarto considera ambas as incertezas.

\section{Procedimento F1}

(i) $\mathrm{R}^{*}=$ inteiro mais próximo de $\left(2 \cdot \mathrm{c}_{1} /\left(\mathrm{c}_{3} \cdot \mathrm{d} \mathrm{m}(\mathrm{T}=0)\right)^{1 / 2}\right.$ (NADDOR, 1975),

$\mathrm{R}^{*}$ é o intervalo ótimo entre reposições

$\mathrm{L}^{*}$ = inteiro maior ou igual ao lead-time médio

(ii) se $t=t_{p}=N \cdot R^{*}$ (N natural, definindo os momentos de execução de

$$
\begin{aligned}
& \text { pedidos, } \mathrm{t}_{\mathrm{p}} \text { ), então: }
\end{aligned}
$$

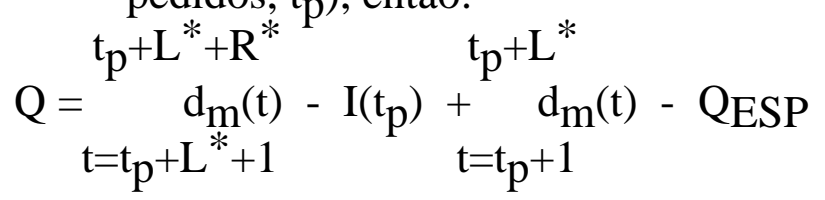

ou seja, deve-se pedir o suficiente para (1) atender à soma das projeções de demanda referentes ao intervalo ótimo entre reposições após $L^{*}$ (intervalo entre $L^{*}$ e $L^{*}+R^{*}$, até que um novo pedido possa chegar e atender períodos subseqüentes) e (2) corrigir distorções devidas a possíveis diferenças entre as quantidades solicitadas e não entregues e as demandas ocorridas no intervalo anterior, descontado o estoque disponível inicial. 


\section{Procedimento F2}

(i) $\mathrm{R}^{*}=$ inteiro mais próximo de $\left(2 \cdot \mathrm{c}_{1} /\left(\mathrm{c}_{3} \cdot \mathrm{d}_{\mathrm{m}}(\mathrm{T}=0)\right)^{1 / 2}\right.$

$\mathrm{L}^{*}=$ inteiro maior ou igual ao lead-time médio

(ii) $\mathrm{A}=\mathrm{c}_{2} /\left(\mathrm{c}_{2}+\mathrm{c}_{3}\right), \quad \mathrm{F}_{\mathrm{T}}(\mathrm{k})=\mathrm{A}$

(iii) se $t=t_{p}=N \cdot R^{*}$, então:

$$
\mathrm{Q} \underset{\mathrm{t}=\mathrm{t}_{\mathrm{p}}+\mathrm{L}^{*+1}}{=}\left(\mathrm{d}_{\mathrm{m}}(\mathrm{t})+\mathrm{k} .(\mathrm{t})\right)-\mathrm{I}\left(\mathrm{t}_{\mathrm{p}}\right)+\underset{\mathrm{t}=\mathrm{t}_{\mathrm{p}}+1}{\mathrm{t}_{\mathrm{p}}+\mathrm{L}^{*}+\mathrm{L}^{*}}(\mathrm{t})-\mathrm{QESP}
$$

Neste caso, há uma margem de segurança para atender demandas acima do valor médio projetado.

\section{Procedimento F3}

(i) $\mathrm{R}^{*}=$ inteiro mais próximo de $\left(2 \cdot \mathrm{c}_{1} /\left(\mathrm{c}_{3} \cdot \mathrm{d}_{\mathrm{m}}(\mathrm{T}=0)\right)^{1 / 2}\right.$

$\mathrm{L}^{*}=$ inteiro maior ou igual ao lead-time máximo

(ii) se $\mathrm{t}=\mathrm{t}_{\mathrm{p}}=\mathrm{N} \cdot \mathrm{R}^{*}$, então:

$$
\mathrm{Q}=\underset{\mathrm{t}=\mathrm{t}_{\mathrm{p}}+\mathrm{L}^{*}+1}{\mathrm{~d}_{\mathrm{m}}(\mathrm{t})-\mathrm{I}\left(\mathrm{t}_{\mathrm{p}}\right)}+\underset{\mathrm{t}=\mathrm{t}_{\mathrm{p}}+1}{\mathrm{t}_{\mathrm{p}}+\mathrm{L}^{*}+\mathrm{L}^{*}}
$$

\section{Procedimento F4}

(i) $\mathrm{R}^{*}=$ inteiro mais próximo de $\left(2 \cdot \mathrm{c}_{1} /\left(\mathrm{c}_{3} \cdot \mathrm{d}_{\mathrm{m}}(\mathrm{T}=0)\right)^{1 / 2}\right.$

$\mathrm{L}^{*}=$ inteiro maior ou igual ao lead-time máximo

(ii) $\mathrm{A}=\mathrm{c}_{2} /\left(\mathrm{c}_{2}+\mathrm{c}_{3}\right), \quad \mathrm{F}_{\mathrm{T}}(\mathrm{k})=\mathrm{A}$

(iii) se $t=t_{p}=N \cdot R^{*}$, então

$$
\underset{\mathrm{t}=\mathrm{t}_{\mathrm{p}}+\mathrm{L}^{*}+1}{\stackrel{\mathrm{t}_{\mathrm{p}}}{=}\left(\mathrm{L}_{\mathrm{m}}(\mathrm{t})+\mathrm{k} .(\mathrm{t})\right)-\mathrm{I}\left(\mathrm{t}_{\mathrm{p}}\right) \stackrel{\mathrm{T}^{*}}{\mathrm{t}_{\mathrm{p}}+\mathrm{L}^{*}} \mathrm{~d}_{\mathrm{m}=\mathrm{t}_{\mathrm{p}}}(\mathrm{t})-\mathrm{Q}_{\mathrm{ESP}}}
$$

Deve-se ressaltar que os custos unitários não terão modificações ao longo do tempo. Além dos modelos apresentados por muitos autores para mudanças instantâneas do custo do material, LEV \& WEISS (1988) discutem problemas com mudanças instantâneas de quaisquer custos.

A tabela a seguir resume as hipóteses adotadas nos procedimentos que serão avaliados no estudo:

Tabela 1 - Caracterização dos Procedimentos a Serem Avaliados 


\begin{tabular}{|c|c|c|c|c|c|c|c|}
\hline \multirow[b]{2}{*}{$\mathrm{PROC}$} & \multicolumn{7}{|c|}{ HIPÓTESE } \\
\hline & POLÍTICA & REVISÃO & CERTEZA & \begin{tabular}{|l} 
COMPORT. \\
\end{tabular} & LEAD-TIME & PEDIDO & HORIZONTE \\
\hline A1 & $(\mathrm{s}, \mathrm{Q})$ & CONTÍNUA & DETERM. & ESTÁVEL & DETERM. & FIXO & INFINITO \\
\hline A2 & $(\mathrm{s}, \mathrm{Z})$ & CONTÍNUA & DETERM. & ESTÁVEL & DETERM. & VARIÁVEL & INFINITO \\
\hline B1 & $(\mathrm{s}, \mathrm{Q})$ & CONTÍNUA & PROBAB. & ESTÁVEL & DETERM. & FIXO & INFINITO \\
\hline B2 & (Z) & CONTÍNUA & PROBAB. & ESTÁVEL & DETERM. & VARIÁVEL & INFINITO \\
\hline B3 & $(\mathrm{s}, \mathrm{Q})$ & CONTÍNUA & PROBAB. & ESTÁVEL & DETERM. & FIXO & INFINITO \\
\hline B4 & $(\mathrm{s}, \mathrm{Q})$ & CONTÍNUA & PROBAB. & ESTÁVEL & DETERM. & FIXO & INFINITO \\
\hline B5 & $(\mathrm{s}, \mathrm{Q})$ & CONTÍNUA & PROBAB. & ESTÁVEL & DETERM. & FIXO & INFINITO \\
\hline B6 & $(\mathrm{s}, \mathrm{Q})$ & CONTÍNUA & PROBAB. & ESTÁVEL & DETERM. & FIXO & INFINITO \\
\hline $\mathrm{C} 1$ & $(\mathrm{~s}, \mathrm{Z}) /(\mathrm{Z})$ & PERIÓDICA & PROBAB. & ESTÁVEL & DETERM. & VARIÁVEL & INFINITO \\
\hline $\mathrm{C} 2$ & $(s, Z)$ & PERIÓDICA & PROBAB. & ESTÁVEL & DETERM. & VARIÁVEL & INFINITO \\
\hline $\mathrm{C} 3$ & $(\mathrm{R}, \mathrm{Z})$ & PERIÓDICA & PROBAB. & ESTÁVEL & DETERM. & VARIÁVEL & INFINITO \\
\hline $\mathrm{C} 4$ & $(\mathrm{R}, \mathrm{s}, \mathrm{Z})$ & PERIÓDICA & PROBAB. & ESTÁVEL & DETERM. & VARIÁVEL & INFINITO \\
\hline D1 & $(\mathrm{s}, \mathrm{Q})$ & CONTÍNUA & DETERM. & CRESCENTE & DETERM. & FIXO & FINITO \\
\hline D2 & $(\mathrm{s}, \mathrm{Q})$ & CONTÍNUA & DETERM. & CRESCENTE & DETERM. & FIXO & FINITO \\
\hline E1 & $(\mathrm{s}, \mathrm{Q})$ & \begin{tabular}{|l|} 
CONT/PER \\
\end{tabular} & PROBAB. & ESTÁVEL & PROBAB. & FIXO & INFINITO \\
\hline E2 & $(\mathrm{s}, \mathrm{Q})$ & \begin{tabular}{|l|l|} 
CONT/PER \\
\end{tabular} & PROBAB. & ESTÁVEL & PROBAB. & FIXO & INFINITO \\
\hline F1 & $(R, Q)$ & PERIÓDICA & DETERM. & \begin{tabular}{|l|l|} 
VARIÁVEL \\
\end{tabular} & DETERM. & VARIÁVEL & INFINITO \\
\hline F2 & $(\mathrm{R}, \mathrm{Q})$ & PERIÓDICA & PROBAB. & VARIÁVEL & DETERM. & VARIÁVEL & INFINITO \\
\hline F3 & $(\mathrm{R}, \mathrm{Q})$ & PERIÓDICA & DETERM. & VARIÁVEL & PROBAB. & VARIÁVEL & INFINITO \\
\hline F4 & $(\mathrm{R}, \mathrm{Q})$ & PERIÓDICA & PROBAB. & VARIÁVEL & PROBAB. & VARIÁVEL & INFINITO \\
\hline
\end{tabular}

\section{O Modelo de Avaliação de Procedimentos}

Além da hipótese de total perda de venda quando a demanda não é atendida, as seguintes hipóteses serão adotadas no estudo:

- os bens são tratados independentemente e não são perecíveis no curto prazo;

- não há a inversão de ordens de compra e os pedidos chegam no início da semana;

- os custos e receitas unitários são constantes ao longo do tempo;

- os custos e receitas são incorridos nos seguintes instantes: custo de pedido no final da semana em que é feito; custo de material, no final da semana de chegada do bem; custos de estocagem e falta, no final da semana da ocorrência; receita de venda; no final da semana de venda;

- não há situações de desconto nos preços em função da quantidade adquirida;

- as quantidades envolvidas são variáveis inteiras;

- a taxa de fornecimento dos bens é infinita;

- os custos de operação do sistema de informações são independentes do sistema de gestão e da operação física da empresa;

- a dispersão da demanda é conhecida e constante (mediante a sua variação percentual);

- o comportamento da demanda (distribuição e coeficientes de crescimento e sazonalidade) não é conhecido pelos procedimentos. Para definir seus parâmetros, utiliza-se um módulo de projeção, que funcionará baseado num comportamento inicial (conhecido) e nas demandas reais (geradas aleatoriamente em torno de um comportamento médio definido).A demanda foi gerada por meio da fórmula abaixo: 


$$
\begin{aligned}
\mathrm{X}(\mathrm{t})= & \mathrm{X}_{\mathrm{m}}(\mathrm{t})+\cdot(\mathrm{t}) \text {, onde: } \\
\mathrm{X}(\mathrm{t})= & \text { demanda real do período } \mathrm{t} \\
\mathrm{X}_{\mathrm{m}}(\mathrm{t})= & \text { demanda média do período } \mathrm{t} \\
& =\text { função de distribuição normal (média } 0 \text { e desvio-padrão } 1) \\
(\mathrm{t})= & \text { desvio-padrão da demanda no período t, igual a VAR . } \mathrm{X}_{\mathrm{m}}(\mathrm{t}), \\
& \text { VAR um indicador (percentual) da aleatoriedade da demanda } \\
& \text { Os valores de } \mathrm{X}_{\mathrm{m}}(\mathrm{t}) \text { são obtidos a partir de: } \\
& \mathrm{X}_{\mathrm{m}}(\mathrm{t})=\mathrm{F}_{\mathrm{p}}(\mathrm{t}) \cdot(\mathrm{S}(0)+\mathrm{R}(0) \cdot \mathrm{t}) \text {, onde: } \\
& \mathrm{S}(0)=\text { valor inicial da demanda (instante } \mathrm{t}=0 \text { ) } \\
& \mathrm{R}(0)=\text { valor inicial da tendência de crescimento da demanda } \\
& \mathrm{F}_{\mathrm{p}}(\mathrm{t})=\text { fator de sazonalidade para o período } \mathrm{t} \text { (que se repete } \\
& \text { para os ciclos seguintes) }
\end{aligned}
$$

A projeção da demanda é baseada na seguinte fórmula:

$$
\begin{aligned}
& \operatorname{PROJ}(\mathrm{t})=\mathrm{F}_{\mathrm{p}}(\mathrm{t}) \cdot\left(\mathrm{S}\left(\mathrm{t}_{0}\right)+\left(\mathrm{t}-\mathrm{t}_{0}\right) \cdot \mathrm{R}\left(\mathrm{t}_{0}\right)\right) \text {, onde: } \\
& \operatorname{PROJ}(\mathrm{t})= \text { demanda projetada para o período } \mathrm{t} \text { (período posterior ao } \\
&\text { último período cuja demanda real é conhecida, } \left.\mathrm{t}_{0}\right) \\
& \mathrm{S}\left(\mathrm{t}_{0}\right) \quad=\text { valor médio da tendência verificada até a semana anterior } \\
& \mathrm{R}\left(\mathrm{t}_{0}\right) \quad=\text { inclinação da curva de demanda na semana anterior } \\
& \mathrm{F}_{\mathrm{p}}(\mathrm{t}) \quad=\text { fator de sazonalidade para o período em questão }
\end{aligned}
$$

A atualização dos parâmetros para projeção é feita seguindo os passos abaixo:

$$
\begin{aligned}
& \mathrm{S}\left(\mathrm{t}_{0}\right)=\mathrm{a} \cdot\left(\mathrm{X}\left(\mathrm{t}_{0}\right)\right) / \mathrm{F}_{\mathrm{p}}\left(\mathrm{t}_{0}\right)+(1-\mathrm{a}) \cdot\left(\mathrm{PROJ}\left(\mathrm{t}_{0}\right)\right) / \mathrm{F}_{\mathrm{p}}\left(\mathrm{t}_{0}\right) \\
& \mathrm{R}\left(\mathrm{t}_{0}\right)=\mathrm{b} \cdot\left(\mathrm{S}\left(\mathrm{t}_{0}\right)-\mathrm{S}\left(\mathrm{t}_{0}-1\right)\right)+(1-\mathrm{b}) \cdot \mathrm{R}\left(\mathrm{t}_{0}-1\right) \\
& \mathrm{F}_{\mathrm{p}}\left(\mathrm{t}_{0}+\mathrm{P}\right)=\mathrm{c} \cdot\left(\mathrm{X}\left(\mathrm{t}_{0}\right)\right) / \mathrm{S}\left(\mathrm{t}_{0}\right)+(1-\mathrm{c}) \cdot \mathrm{F}_{\mathrm{p}}\left(\mathrm{t}_{0}\right)
\end{aligned}
$$

A opção pelo suavizamento exponencial para a previsão da demanda baseia-se no fato de que o comportamento desta é considerado como dependente única e exclusivamente da variável independente tempo. Além disto, optou-se pela abordagem (e, em consequência, pelo uso das respectivas fórmulas) proposta por Holt e Winters (apud GARDNER, 1985). Segundo GARDNER, tal abordagem é simples no seu entendimento e manipulação, além de proporcionar resultados tão ou mais satisfatórios que outras técnicas de projeção disponíveis (Box-Jenkins, séries de Fourier, etc).

O modelo de avaliação proposto para avaliar os procedimentos de planejamento e controle de estoques consiste num programa de simulação (esquematizado na figura 1), que realiza a avaliação física e financeira de cada procedimento, a partir do conhecimento do comportamento da demanda ao longo do tempo, que foi gerada por meio de um programa auxiliar. Os programas foram escritos na linguagem Turbo Pascal 5.5 e as simulações realizadas num microcomputador DX 386, com freqüência de $40 \mathrm{MHz}$ e RAM de 4 Mbytes. 
LEITURA RECEITA, CUSTOS UNITÁRIOS, COEFICIENTES DE SUAVIZAMENTO, INTERVALO ENTRE PEDIDOS E LOTE MÍNIMOS, LOTE-PADRÃO DEMANDA (DADOS INICIAIS PARA COMEÇAR PROJEÇÕES; DEMANDAS REAIS)

$\mathrm{T}=1$

CÁLCULOS NO PERÍODO (ESTOQUES/FALTAS, CUSTOS DE MATERIAL, FALTAS E ESTOCAGEM, RECEITA E MARGEM PRELIMINAR (SEM CUSTO DE PEDIDO))

PROJEÇÃO DA DEMANDA/ATUALIZAÇÃO PARÂMETROS

CÁLCULO DOS PARÂMETROS DO PROCEDIMENTO

NÃO

EXECUTA-SE

O PEDIDO ?

SIM

CÁLCULO PRECISO DO PEDIDO, DO CUSTO DO PEDIDO E DA MARGEM FINAL

$\mathrm{T}=\mathrm{T}+1$

NÃO

T> TFIM ?

SIM

CÁLCULOS FINAIS (MARGENS MÉDIA, MÁXIMA E MINIMA, CUSTOS MÉDIOS DE MATERIAL, PEDIDO,FALTA, ESTOCAGEM E TOTAL, ESTOQUES E FALTAS MÉDIOS E MÁXIMOS, PERCENTUAL DE SEMANAS COM ESTOQUES E COM FALTAS, INTERVALO MÉDIO ENTRE PEDIDOS, PEDIDOS MÉDIO, MÁXIMO E MÍNIMO)

Figura 1 - Fluxograma do Programa de Simulação

\section{Ambientes da Empresa}

Os valores apresentados para os parâmetros de entrada (extrapolados a partir de PORTEUS, 1985) procuram representar diferentes ordens de grandeza entre eles.

Os fatores iniciais de sazonalidade, para cada hipótese, têm os seguintes valores:

nula: $\quad \mathrm{t}$ de 1 a $52, \mathrm{FP}_{\mathrm{P}}(\mathrm{t})=1$

senoidal anual: $\mathrm{t}$ de 1 a $52, \mathrm{~F}_{\mathrm{P}}(\mathrm{t})=(1+0,5 \cdot \operatorname{sen}(2$. .t/52) $)$

pontual: $\quad t$ de 1 a 47,51 e $52, F_{P}(t)=1$; $t$ de $48 a 50, F_{P}(t)=3$ 
A tabela 3 apresenta as 40 situações propostas para análise.

Tabela 2 - Condições dos Ambientes da Empresa

\begin{tabular}{|c|c|c|c|c|c|c|c|c|c|}
\hline \multirow[b]{2}{*}{ AMBIENTE } & \multicolumn{9}{|c|}{ PARÂMETRO } \\
\hline & $\mathrm{c} 1$ & C5 & $\mathrm{RU}$ & $S(0)$ & $(0) / S(0)$ & $\mathrm{R}(0) / \mathrm{S}(0)$ & Sazonalidade & $\mathrm{LT}_{\text {mín- }} \mathrm{LT}_{\text {máx }}$ & a,b,c \\
\hline 1 & 100 & 10 & 200 & 200 & $5 \%$ & $0 \%$ & NULA & $2-2$ & 0,1 \\
\hline 2 & 100 & 10 & 200 & 200 & $5 \%$ & $0 \%$ & NULA & $2-2$ & 0,3 \\
\hline 3 & 100 & 10 & 200 & 200 & $5 \%$ & $0 \%$ & NULA & $1-3$ & 0,1 \\
\hline 4 & 10 & 10 & 200 & 200 & $5 \%$ & $0 \%$ & NULA & $2-2$ & 0,1 \\
\hline 5 & 1 & 10 & 200 & 200 & $5 \%$ & $0 \%$ & NULA & $2-2$ & 0,1 \\
\hline 6 & 100 & 100 & 200 & 200 & $5 \%$ & $0 \%$ & NULA & $2-2$ & 0,1 \\
\hline $7 *$ & 100 & 10 & 200 & 200 & $5 \%$ & $0 \%$ & NULA & $2-2$ & 0,1 \\
\hline $8 * *$ & 100 & 10 & 200 & 200 & $5 \%$ & $0 \%$ & NULA & $2-2$ & 0,1 \\
\hline 9 & 100 & 10 & 200 & 200 & $10 \%$ & $0 \%$ & NULA & $2-2$ & 0,1 \\
\hline 10 & 100 & 10 & 200 & 200 & $10 \%$ & $0 \%$ & NULA & $1-3$ & 0,1 \\
\hline 11 & 100 & 10 & 200 & 200 & $10 \%$ & $0 \%$ & NULA & $2-2$ & 0,3 \\
\hline 12 & 100 & 10 & 200 & 200 & $5 \%$ & $0 \%$ & ANUAL & $2-2$ & 0,1 \\
\hline 13 & 100 & 10 & 200 & 200 & $5 \%$ & $0 \%$ & ANUAL & $1-3$ & 0,1 \\
\hline 14 & 100 & 10 & 200 & 200 & $10 \%$ & $0 \%$ & ANUAL & $2-2$ & 0,1 \\
\hline 15 & 100 & 10 & 200 & 200 & $5 \%$ & $0 \%$ & PONTUAL & $2-2$ & 0,1 \\
\hline 16 & 100 & 10 & 200 & 200 & $5 \%$ & $0 \%$ & PONTUAL & $1-3$ & 0,1 \\
\hline 17 & 100 & 10 & 200 & 200 & $5 \%$ & $0 \%$ & NULA & $4-4$ & 0,1 \\
\hline 18 & 100 & 10 & 200 & 20 & $5 \%$ & $0 \%$ & NULA & $2-2$ & 0,1 \\
\hline 19 & 100 & 10 & 200 & 20 & $5 \%$ & $0 \%$ & ANUAL & $2-2$ & 0,1 \\
\hline 20 & 100 & 10 & 200 & 20 & $5 \%$ & $0 \%$ & PONTUAL & $2-2$ & 0,1 \\
\hline 21 & 100 & 10 & 200 & 200 & $5 \%$ & $0,35 \%$ & NULA & $2-2$ & 0,1 \\
\hline 22 & 100 & 10 & 200 & 200 & $5 \%$ & $0,35 \%$ & NULA & $2-2$ & 0,3 \\
\hline 23 & 100 & 10 & 200 & 200 & $5 \%$ & $0,35 \%$ & NULA & $1-3$ & 0,1 \\
\hline 24 & 10 & 10 & 200 & 200 & $5 \%$ & $0,35 \%$ & NULA & $2-2$ & 0,1 \\
\hline 25 & 100 & 10 & 200 & 200 & $5 \%$ & $0,35 \%$ & ANUAL & $2-2$ & 0,1 \\
\hline 26 & 100 & 10 & 200 & 200 & $5 \%$ & $0,35 \%$ & ANUAL & $2-2$ & 0,3 \\
\hline 27 & 100 & 10 & 200 & 200 & $5 \%$ & $0,35 \%$ & ANUAL & $1-3$ & 0,1 \\
\hline 28 & 100 & 10 & 200 & 200 & $5 \%$ & $0,35 \%$ & PONTUAL & $2-2$ & 0,1 \\
\hline 29 & 100 & 10 & 200 & 200 & $5 \%$ & $0,78 \%$ & NULA & $2-2$ & 0,1 \\
\hline 30 & 100 & 10 & 200 & 200 & $5 \%$ & $0,78 \%$ & ANUAL & $2-2$ & 0,1 \\
\hline 31 & 100 & 10 & 200 & 200 & $5 \%$ & $-0,35 \%$ & NULA & $2-2$ & 0,1 \\
\hline 32 & 100 & 10 & 200 & 200 & $5 \%$ & $-0,35 \%$ & ANUAL & $2-2$ & 0,1 \\
\hline 33 & 100 & 0 & 120 & 200 & $5 \%$ & $0 \%$ & NULA & $2-2$ & 0,1 \\
\hline 34 & 100 & 10 & 200 & 500 & $5 \%$ & $0 \%$ & NULA & $2-2$ & 0,1 \\
\hline 35 & 50 & 10 & 200 & 500 & $5 \%$ & $0 \%$ & NULA & $1-3$ & 0,1 \\
\hline 36 & 10 & 0 & 120 & 200 & $5 \%$ & $0 \%$ & NULA & $2-2$ & 0,1 \\
\hline 37 & 10 & 0 & 120 & 200 & $5 \%$ & $0 \%$ & NULA & $1-3$ & 0,1 \\
\hline 38 & 50 & 10 & 150 & 200 & $5 \%$ & $0,35 \%$ & ANUAL & $2-2$ & 0,1 \\
\hline 39 & 100 & 10 & 200 & 500 & $5 \%$ & $0,35 \%$ & ANUAL & $1-3$ & 0,1 \\
\hline 40 & 10 & 0 & 120 & 500 & $5 \%$ & $0,35 \%$ & ANUAL & $1-3$ & 0,1 \\
\hline
\end{tabular}

Notação da tabela 2:

* = periodicidade mínima de 4 semanas entre pedidos ** = pedido múltiplo de 12

$\mathrm{c}_{1}=$ custo unitário de pedido

$\mathrm{RU}=$ receita unitária

$(0) / S(0)=$ aleatoriedade da demanda

$\mathrm{C}_{5}$ = custo unitário de falta, além da perda de venda

Sazonalidade $=$ tipo de sazonalidade $\mathrm{d}$ $\mathrm{a}, \mathrm{b}, \mathrm{c}=$ coeficientes de suavizamento

$\mathrm{S}(0)=$ demanda média inicial

$\mathrm{R}(0) / \mathrm{S}(0)=$ taxa de crescimento da demanda

$\mathrm{LT}_{\text {mín }}-\mathrm{LT}_{\text {máx }}=$ lead-times mínimo e máximo 
Os parâmetros fixos que compõem os ambientes são: custo de material (c4 $=100$ $\$$ /unidade); custo de estocagem (c3 $=1 \$$ /unidade/semana); taxa de juros (j' $=0,5$ $\% /$ semana); estoque inicial (IIF(0) igual à demanda média inicial); período total (TFIM = 530 semanas) e o período inicial, a ser desconsiderado, de 10 semanas.

\section{Resultados e Conclusões}

Para cada um dos 32 primeiros ambientes descritos no item 5, os quais caracterizam diferentes situações de mercado (e, em alguns casos, diferentes coeficientes de suavizamento para atualização das projeções), foram testados todos os 20 procedimentos selecionados e descritos no item 3. Para cada simulação, a entrada de dados (via teclado) consumiu, em média, $15 \mathrm{~s}$, mesmo tempo gasto pelo microcomputador para executar cada simulação. A impressão dos resultados consumiu em torno de 30s para cada simulação.

Posteriormente, alguns procedimentos (B2, C1, E2 e F4, escolhidos por apresentarem desempenho superiore ao dos demais, das respectivas famílias, e muito superior ao daqueles das famílias A e D), foram testados em ambientes semelhantes aos anteriores, apenas variando-se alguns dos valores que os caracterizam (por exemplo, alteração da demanda média de 200 para 500, ou alteração do custo de pedido de 100 para 50), com o intuito de verificar se o desempenho relativo dos procedimentos seria mantido (ou seja, se um melhor desempenho não seria decorrente de valores muito particulares do ambiente).

Finalmente, para o procedimento $\mathrm{C} 1$, inicialmente desenvolvido para considerar o lead-time médio para a definição do nível de reposição s (como os demais da família C), foi feita uma última alteração, não apresentada no item 3 , que seria a consideração do lead-time máximo para a definição deste parâmetro.

As tabelas 3 e 4 apresentam, respectivamente, os resultados das simulações com relação a dois indicadores de desempenho; a primeira tabela apresenta, para cada ambiente, o desempenho relativo de cada procedimento frente ao desempenho do melhor procedimento no ambiente, em termos de margem média obtida no período de simulação (descontadas as dez semanas iniciais), enquanto a segunda apresenta 0 percentual de demanda não atendida pelos procedimentos em cada ambiente, no mesmo horizonte de tempo.

As simulações realizadas permitem concluir que os procedimentos baseados em modelos de revisão periódica (nos quais há a hipótese de que a empresa executa o apontamento dos níveis de estoques e gera pedidos em intervalos discretos de tempo) são mais adequados do que os baseados em revisão contínua (apontamento contínuo e geração do pedido a qualquer momento em que se faça necessário), da mesma forma como são mais eficazes os procedimentos que consideram demanda probabilística com relação aos que operam com demandas determinísticas (nas quais os valores adotados são as médias das distribuições probabilísticas).

Um tratamento estatístico razoavelmente simples pode ser incorporado aos procedimentos para que estes tenham um certo grau de segurança com relação à aleatoriedade da demanda, de forma a induzir os sistemas a trabalharem com um maior 
estoque médio, diminuindo a incidência de faltas (esta segurança deve ser maior quanto maior for o custo relativo da perda de venda, frente ao custo de estocagem).

Nos casos em que o lead-time de entrega dos bens é probabilístico, verificou-se ser mais vantajoso calcular os parâmetros dos procedimentos com base nos valores máximos do que nos valores médios (ao menos para distribuições do lead-time semelhantes à considerada neste estudo - uniforme e com pequena diferença entre valores máximos e mínimos. Para distribuições semelhantes à normal, o valor mais adequado certamente seria superior ao valor médio e inferior ao valor máximo).

Menores custos relativos de pedido nem sempre puderam ser aproveitados significativamente porque o menor intervalo permitido entre pedidos era de 1 semana. Por sua vez, a variação do custo relativo de falta afetou bastante os procedimentos que não o levam em consideração (produzindo resultados físicos iguais), fato não ocorrido com os de maior eficácia (que consideram o custo de falta para definir seus parâmetros).

Diferentes níveis de demanda podem alterar a diferença de desempenho entre os procedimentos mais eficazes e os menos eficazes (com base no percentual de margem média obtida com relação ao melhor procedimento), embora os melhores sejam os mesmos em qualquer uma das situações. A mesma conclusão pode ser tomada com relação a diferentes taxas de crescimento da demanda e diferentes valores de leadtime.

Utilizando-se modelos de projeção de demanda baseados em médias móveis exponenciais, a adoção de diferentes coeficientes de suavizamento para a atualização das projeções não levou a grandes variações nos resultados alcançados pelos procedimentos.

Os desempenhos relativos entre os procedimentos avaliados neste estudo não foram alterados em função de diferentes aleatoriedades da demanda. Para maiores aleatoriedades, os valores de falta média não foram significativamente diferentes dos encontrados em situações de menor aleatoriedade (nas quais as demandas reais são relativamente mais próximas às projetadas).

Os procedimentos que operam baseados na projeção da demanda de vários períodos à frente (os baseados no cálculo de necessidades) são os mais indicados para situações em que a demanda apresenta alterações significativas no curto prazo. Para as demais situações (demanda estável, crescente ou decrescente, sem sazonalidade ou com sazonalidade suave no curto prazo) os procedimentos baseados em revisão períódica produzem resultados tão ou mais satisfatórios do que os baseados no cálculo de necessidades.

O presente estudo poderia ser estendido de várias formas. Como extensões mais óbvias, haveria a extrapolação do exercício para outros procedimentos existentes na literatura e para outros ambientes, de forma a obter resultados mais generalizáveis.

Com relação aos procedimentos testados neste estudo, poderiam ser feitas, ainda, alterações com relação à consideração de lead-times máximos (ao invés de médios) e de demandas probabilísticas (ao invés de determinísticas) em alguns procedimentos. 
Tais alterações não foram testadas neste estudo para não descaracterizar totalmente os procedimentos, de forma a permitir a análise do desempenho de diversos tipos de procedimentos (com suas hipóteses particulares). Especificamente com relação aos procedimentos da família $\mathrm{F}$, poderia ser incluído um passo que permitisse alterar o intervalo entre reposições, tornando os procedimentos mais eficazes em ambientes com alterações de demanda e de relações entre os custos. Também poderiam ser testadas outras formas de cálculo do coeficiente de segurança, para a demanda $\mathrm{k}$.

Poderiam, ainda, ser incorporadas outras formas de previsão de demanda, com o intuito de analisar o desempenho relativo entre as várias combinações de procedimentos e métodos de previsão (ainda utilizando suavizamento exponencial, poderia ser testada a utilização da projeção da demanda para o período em que, na média, seria esperado o pedido que estaria sendo efetuado. Este "avanço" somente foi incorporado aos procedimentos da família F, pois os demais partiam da suposição de que a demanda seria estável e/ou não havia lead-time. Esta alteração, provavelmente, melhoraria o desempenho destes procedimentos nos ambientes em que houvesse mudanças bruscas na demanda).

Também haveria utilidade em se verificar o comportamento dos procedimentos em ambientes com alteração (contínua ou esporádica) da receita e dos custos unitários (incluindo ambientes com inflação), além de situações em que há descontos em função da quantidade adquirida.

Ainda restrito à situação de perda de venda, poderiam ser propostos procedimentos que permitissem a análise em ambientes com vários produtos, incluindo restrições de espaço e financeiras, por exemplo.

Finalmente, processo semelhante poderia ser utilizado para avaliar procedimentos para operar em situações nas quais é possível o atraso na entrega dos bens e situações intermediárias entre esta e a perda total da demanda não atendida no momento da procura pelo cliente, além de situações em que os bens tratados sejam perecíveis.

\section{Referências Bibliográficas:}

AMRANI, M. \& RAND, G.K.: "An ecletic algorithm for inventory replenishment for items with increasing linear trend in demand". Engineering Costs and Production Economics, v.19, p.261-266, 1990.

DAS, C.: "On the use of a structural equation for determining inventory order quantities". Naval Research Logistics Quarterly, v.32, n.4, p.689-692, 1985.

DAVE, U.: "A deterministic lot-size inventory model with shortages and a linear trend in demand". Naval Research Logistics, v.36, n.4, p.507-514, 1989a.

DAVE, U.: "On a heuristic inventory-replenishment rule for items with linearly increasing demand incorporating shortages". Journal of the Operational Research Society, v.40, n.9, p.827-830, 1989b. 
FORDYCE, J.M. \& WEBSTER, F.M.: "The Wagner-Whitin algorithm made simple". Production and Inventory Management, v.25, n.2, p.21-30, 1984.

FRIEDMAN, M.F.: "Power-form demand and cost functions in inventory lot size models". Computers \& Operations Research, v.8, n.3, p.159-167, 1981.

FRIEDMAN, M.F.: "The effect of stochastic supply times on optimal policies in inventory control". Engineering Costs and Production Economics, v.15, p.329333, 1988.

GARDNER JR., E.S.: "Exponential smoothing: the state of the art". Journal of Forecasting, v.4, p.1-28, 1985.

HAX, A.; CANDEA, D.: Production and inventory management. Englewood Cliffs: N.J., Prentice Hall, 1984.

HENERY, R.J.: "Inventory replenishment policy for cyclic demand". Journal of the Operational Research Society, v.41, n.7, p.639-643, 1990.

LEV, B. \& WEISS, H.J.: "Inventory models with cost changes". Operations Research, v.38, n.1, p.53-63, 1990.

LOVE, S.F.: Inventory control. McGraw-Hill: New York, 1979.

MABIN, V.J.: "A practical near-optimal order quantity method". Engineering Costs and Production Economics, v.15, p.381-386, 1988.

MURDESHWAR, T.M.: "Inventory replenishment policy for linearly increasing demand considering shortages - An optimal solution". Journal of the Operational Research Society, v.39, n.7, p.687-692, 1988.

NADDOR, E.: "Optimal and heuristic decisions in single and multi-item inventory systems". Management Science, v.21, n.11, p.1234-1249, 1975.

PETERSON, R. \& SILVER, E.A.: Decision systems for inventory management and production control. John Wiley \& Sons: New York, 1985.

PORTEUS, E.L.: "Numerical comparisons of inventory policies for periodic review systems". Operations Research, v.33, n.1, p.134-152, 1985.

RITCHIE, E.: "The EOQ for linear increasing demand: A simple optimal solution". Journal of the Operational Research Society, v.35, n.10, p.949-952, 1984.

RITCHIE, E. \& TSADO, A.K.: "Stock replenishment quantities for unbounded linear increasing demand: An interesting consequence of the optimal policy". Journal of the Operational Research Society, v.36, n.8, p.737-739, 1985.

SNYDER, R.D.: "Computation of (S,s) ordering policy parameters". Management Science, v.21, n.2, p.223-229, 1974.

SPHICAS, G. \& NASRI, F.: "An inventory model with finite-range stochastic lead times". Naval Research Logistics Quarterly, v.31, n.4, p.609-616, 1984.

TSADO, A.K.: "A simple inventory replenishment policy for demand with uncertainty". Journal of the Operational Research Society, v.36, n.11, p.10591068, 1985.

Bibliografia Complementar: 
AARDAL, K.; JONSSON Ö.; JÖNSSON, H.: "Optimal inventory policies with service-level constraints". Journal of the Operational Research Society, v.40, n.1, p.65-73, 1989.

ANDERSON, E.J.: "A note on the dynamic lot-size model with uncertainty in demand and supply processes". Management Science, v.35, n.5, p.635-640, 1989.

ARCELUS, F.J. \& SRINIVASAN, G.: "The sensitivity of optimal inventory policies to model assumptions and parameters". Engineering Costs and Production Economics, v.15, p.291-298, 1988.

ARDALAN, A.: "Optimal ordering policies in response to a sale". Institute of Industrial Engineers Transactions, v.20, n.3, p.292-294, 1988.

AUCAMP, D.: "Separating the cost of capital from the other carrying charges in a discounting formulation of the EOQ problem". International Journal of Operations \& Production Management, v.77, n.6, p.64-70, 1987.

AUCAMP, D. \& KUZDRALL, P.J.: "Order quantities with temporary price reductions". Journal of the Operational Research Society, v.40, n.10, p.937-940, 1989.

AXSÄTER, S.: "Evaluation of lot-sizing techniques". International Journal of Production Research, v.24, n.1, p.27-32, 1986.

BACHMAN, A.: "Inventory control with price flutuations". International Journal of Operations \& Production Management, v.6, n.3, p.58-64, 1986.

BASU, S.K.: "An optimal ordering policy for situations with uncertainty in supply". Naval Research Logistics, v.34, p.151-159, 1987.

BOGATAJ, M. \& BOGATAJ, L.: "Inventory systems optimization for dynamic stochastic and periodical demand". Engineering Costs and Production Economics, v.19, p.295-299, 1990.

BOOKBINDER, J.H. \& TAN, J.: "Strategies for the probabilistic lot-sizing problem with service-level constraints". Management Science, v.34, n.9, p.1096-1108, 1988.

BUFFA, E.S. \& MILLER, J.G.: Production-inventory systems: planning and control. Illinois: Richard D. Irwin Inc., 1979.

BULINSKAYA, E.V.: "Inventory control in case of unknown demand distribution". Engineering Costs and Production Economics, v.19, p.301-306, 1990.

CARLSON, M.L. \& ROSSEAU, J.J.: "EOQ under date-terms supplier credit". Journal of the Operational Research Society, v.40, n.5, p.451-460, 1989.

CHAO, H.: "Inventory policy in the presence of market disruptions". Operations Research, v.35, n.2, p.274-281, 1987.

CHENG, T.C.E.: "Optimal production policy for decaying items with decreasing demand". European Journal of Operational Research, v.43, p.168-173, 1989.

CHOO, E.U. \& CHAN, G.H.: "Eyeballing heuristics for dynamic lot size problems". Computers \& Operations Research, v.16, n.3, p.189-193, 1989.

CHRISTENSEN, B.P. \& FRYMAN, M.A.: "Should we be using cost of capital in calculating holding costs of consumable inventory?". Naval Research Logistics, v.36, p.167-177, 1989. 
CHUNG, K.H.: "Inventory control and trade credit revisited". Journal of the Operational Research Society, v.40, n.5, p.495-498, 1989.

DAS, C.: "On the solution of some approximate continuous review inventory models". Naval Research Logistics Quarterly, v.32, n.2, p.301-313, 1985.

DATTA, T.K. \& PAL, A.K.: "Effects of inflation and time-value of money on an inventory model with linear time-dependent demand rate and shortages". European Journal of Operational Research, v.52, p.326-333, 1991.

DATTA, T.K.; PAL, A.K.: "A note on a replenishment policy for an inventory model with linear trend in demand and shortages". Journal of the Operational Research Society, v.43, n.10, p.993-1001, 1992.

DAVE, U.: "On two deterministic inventory models for items with decreasing demand". Economic Computation and Economic Cybernetics Studies and Research, v.22, n.2, p.45-50, 1987.

DAVE, U.: "On reducing excessive stock in the order-level lot-size system for decaying inventories". Engineering Costs and Production Economics, v.15, p.175-180, 1988.

DOBSON, G.: "Sensitivity of the EOQ model to parameter stimates". Operations Research, v.36, n.4, p.570-574, 1988.

EHRARDT, R.: "Easily computed approximations for $(\mathrm{s}, \mathrm{S})$ inventory system operating characteristics". Naval Research Logistics Quarterly, v.32, n.2, p.347359, 1985.

ELLENRIEDER, A.R.: "Dimensionamento econômico de estoques". Apresentado no 20 Simpósio MOVIMAT, São Paulo, 1983.

ELSAYED, E.A. \& BOUCHER, T.O.: Analysis and control of production systems. Englewood Cliffs: N.J., Prentice Hall, 1985.

FEDERGRUEN, A. \& ZIPKIN, P.: "An efficient algorithm for computing optimal (s,S) policies". Operations Research, v.32, n.6, p.1268-1285, 1984.

GEORGOFF, D.M. \& MURDICK, R.G.: "Manager's guide to forecasting". Harvard Business Review, v.1, p.110-123, 1988.

GERCHAK, Y. \& PARLAR, M.: "Investing in reducing lead-time randomness in continuous-review inventory models". Engineering Costs and Production Economics, v.21, p.191-197, 1991.

GIRLICH, H.: "Naddor's demand patterns and the economic order quantity under uncertainty". Engineering Costs and Production Economics, v.19, p.327-331, 1990.

GLAZER, A. \& HASSIN, R.: "A deterministic single-item inventory model with seller holding cost and buyer holding and shortage costs". Operations Research, v.34, n.4, p.613-618, 1986.

GOLABI, K.: "Optimal inventory policies when ordering prices are random". Operations Research, p.575-588, 1980.

GÜRMANN, K. \& SCHREIBER, K.: "The determination of cost parameters to achieve optimum stockpiling". Engineering Costs and Production Economics, v.19, p.25-30, 1990. 
HWANG, H., MOON, D.H.; SHINN, S.W.: "An EOQ model with quantity discounts for both purchasing price and freight cost". Computers \& Operations Research, v.17, n.1, p.73-78, 1990.

JACOBS, R.A. \& WAGNER, H.M.: "Reducing inventory systems costs by using robust demand stimators". Management Science, v.35, n.7, p.771-787, 1989.

JÖNSSON, H. \& SILVER, E.A.: "Optimal and heuristic solutions for a simple common component inventory problem". Engineering Costs and Production Economics, v.16, p.257-267, 1989.

KIM, Y.H. \& CHUNG, K.H.: "Economic analysis of inventory systems: A clarifying analysis". International Journal of Production Research, v.23, n.4, p.761-767, 1985.

KINGSMAN, B.G. \& BOUSOFIANE, A.: "Ordering and stockpiling under price inflation when prices increase in sucessive discrete jumps". Engineering Costs and Production Economics, v.17, p.395-407, 1989.

LEE, Y.Y. , KRAMER, B.Z.; HWANG, C.L.: "A comparative study of three lotsizing methods for the case of fuzzy demand". International Journal of Operations \& Production Management, v.11, n.7, p.72-80, 1991.

LIU, L.: "(s,S) continuous review models for inventory with random lifetimes". Operations Research Letters, v.9, p.161-167, 1990.

LOVEJOY, W.S.: "Myopic policies for some inventory models with uncertaint demand distributions". Management Science, v.36, n.6, p.724-738, 1990.

LOWE, T.J., SCHWARZ, L.B.; McGAVIN, E.J.: "The determination of optimal basestock inventory policy when the costs of under and oversupply are uncertain". Naval Research Logistics, p.539-554, 1988.

MATTA, K.F.: "Cost function for a periodic reorder-point system with time dependent replenishment costs". European Journal of Operational Research, v.53, p.309316, 1991.

MOINZADEH, K.: "Operating characteristics of the (S-1,S) inventory system with partial backorders and constant resupply times". Management Science, v.35, n.4, p.472-477, 1989.

MYKYTKA, E.F. \& RAMBERG, J.S.: "On the sensitivity of the EOQ to errors in forecast of demand". Institute of Industrial Engineers Transactions, v.16, n.2, p.144-151, 1984.

NADDOR, E.: Inventory systems. Wiley: New York, 1966.

NAHMIAS, S. Approximation techniques for several stochastic inventory models. Computers \& Operations Research, v.8, n.3, p.141-158, 1981.

NTI, K.O.: "Competitive procurement under demand uncertainty". Management Science, v.33, n.11, p.1489-1500, 1987.

PORTEUS, E.L.: "Investing in new parameters values in the discounted EOQ model". Naval Research Logistics Quarterly, v.33, n.1, p.39-48, 1986.

RAMASESH, R.V.: "Procurement under uncertain supply lead-times - A dual sourcing technique could save costs". Engineering Costs and Production Economics, v.21, p.59-68, 1991. 
RATIU-SUCIU, C. \& OPRESCU, G.: "Adjusting the supply process with stochastic models". Economic Computation and Economic Cybernetics Studies and Research, v.21, n.3, p.13-22, 1986.

SHORE, H.: "General approximate solutions for some common inventory models". Journal of the Operational Research Society, v.37, n.6, p.619-629, 1986.

SRINIVASAN, S.K. \& RAVICHANDRAN, N.: "Analysis of (S,s) inventory system with decaying items". Engineering Costs and Production Economics, v.15, p.433-439, 1988.

WALDMANN, K.H.: "Inventory control in randomly varying environments". Society for Industrial and Applied Mathematics Journal on Applied Mathematics, v.44, n.3, p.657-666, 1984.

ZIPKIN, P.: "Critical number policies for inventory models with periodic data". Management Science, v.35, n.1, p.71-80, 1989.

ZIPKIN, P.: "Sthocastic leadtimes in continuous-time inventory models". Naval Research Logistics Quarterly, v.33, n.4, p.763-774, 1986.

ZIPKIN, P.: "Inventory service-level measures: convexity and approximations". Management Science, v.32, n.8, p.975-981, 1986.

\section{INVENTORY MANAGEMENT FOR NON-PERISHABLE RETAIL GOODS}

ABSTRACT - This study compares inventory system management methods for nonperishable retail goods considering the lost sales case. Several methods, based upon models with different assumptions, are tested in environments with characteristics defined on the basis of demand pattern, relations among inventory costs and others. The analysis was made using a simulation software that evaluates the system performance at each period of time in a given time span. The software contains procedures for demand forecasting based upon exponential smoothing, for physical and economic accounting and for determination of the parameters of each management method. The methods based upon periodic review models and probabilistic demand outperformed, respectively, continuous review and deterministic demand models. Besides, the methods that adopt maximun lead-times outperformed those that adopt mean lead-times. The comparative performances of the methods were not affected by changes in demand randomness. The methods that forecast the demand of various periods ahead outperformed the others when there are peaks in demand, but in environments with other demand patterns (steady, increasing or decreasing demand, without seasonality or with smooth seasonality in the short time) similar results were yielded by these methods and those based upon periodic review and steady demand.

Key-words: inventory, simulation, materials management, retail, stock systems 